SUBJECT AREAS:

COMPUTATIONAL NEUROSCIENCE

NEUROSCIENCE

Received

4 March 2014

Accepted

27 June 2014

Published

1 August 2014

Correspondence and requests for materials should be addressed to R.B.P. (rbp@cajal.csic.

\title{
Branching angles of pyramidal cell dendrites follow common geometrical design principles in different cortical
} areas

\author{
Concha Bielza', Ruth Benavides-Piccione ${ }^{2,3}$, Pedro López-Cruz'1 Pedro Larrañaga' \& Javier DeFelipe ${ }^{2,3}$ \\ ${ }^{1}$ Computational Intelligence Group, Departamento de Inteligencia Artificial, Universidad Politécnica de Madrid, Campus de \\ Montegancedo, Boadilla del Monte, 28660 Madrid, Spain, ${ }^{2}$ Instituto Cajal (CSIC), Ave. Dr. Arce, 37, 28002 Madrid, Spain, \\ ${ }^{3}$ Laboratorio Cajal de Circuitos Corticales, Centro de Tecnología Biomédica, Universidad Politécnica de Madrid, Campus de \\ Montegancedo, Pozuelo de Alarcón, 28223 Madrid, Spain.
}

Unraveling pyramidal cell structure is crucial to understanding cortical circuit computations. Although it is well known that pyramidal cell branching structure differs in the various cortical areas, the principles that determine the geometric shapes of these cells are not fully understood. Here we analyzed and modeled with a von Mises distribution the branching angles in 3D reconstructed basal dendritic arbors of hundreds of intracellularly injected cortical pyramidal cells in seven different cortical regions of the frontal, parietal, and occipital cortex of the mouse. We found that, despite the differences in the structure of the pyramidal cells in these distinct functional and cytoarchitectonic cortical areas, there are common design principles that govern the geometry of dendritic branching angles of pyramidal cells in all cortical areas.

M ost synapses in the brain are established with dendrites which represent the main neuronal region specialized to receive and process inputs in neurons. Since there is a great diversity of dendritic morphologies which are specific for each brain region, it follows that there is a large diversity in the design of synaptic circuits. In the cerebral cortex, pyramidal cells are the most common neuron (they constitute approximately $70-85 \%$ of the total population of neurons ${ }^{1}$. These neurons are projection cells and they are located in all layers except layer I. Furthermore, pyramidal cells are the major source of intrinsic excitatory cortical synapses, and their dendritic spines are the main postsynaptic target of excitatory synapses ${ }^{1-6}$. Thus, understanding the principles that determine dendritic geometry of pyramidal cells is essential to comprehend how neuronal circuits operate in the cerebral cortex. Several studies have shown quantitative differences in the size and complexity of the dendritic arbors and in the density and size of dendritic spines in various cortical areas of several rodent and primate species, including humans $\mathrm{s}^{7-13}$. These variations result in fundamental functional differences between the cells, making the study of dendritic properties of considerable interest. For example, the complexity of their dendritic structure determines their biophysical properties, thus influencing their functional capacity and potential for plastic change ${ }^{14-18}$.

In a recent study by our group ${ }^{19}$, a class of probabilistic graphical model called Bayesian networks was applied to model real dendrites and simulate virtual dendrites of the mouse cortex, including primary sensory, motor, and association areas. We observed that terminal segments in basal dendrites were longer than intermediate segments. In fact, dendrites usually branch when they are close to the soma, producing short segments; whereas the segments that do not branch spread away from the soma. Moreover, we showed that segment orientation is mainly controlled by the orientation of the previous segments. Therefore, the dendritic trees tend to first spread rapidly when they are close to the soma and then, once they have reached a minimum size, grow straight away from the soma (see also ref. 20, 21). Furthermore, it has been proposed that branching patterns can be predicted based predominantly on wiring principles and that flatness of 3D dendritic bifurcations (i.e., the condition of a surface where all elements are in one plane) are characteristic of wiring cost optimization ${ }^{22-28}$. However, there are no studies that address possible differences in the branching angle geometry in different cortical areas that would shed light on the branching regional pattern design. Here we analyzed and modeled with a von Mises distribution the branching angles between the two sibling segments growing from a bifurcation in $3 \mathrm{D}$ reconstructed basal 


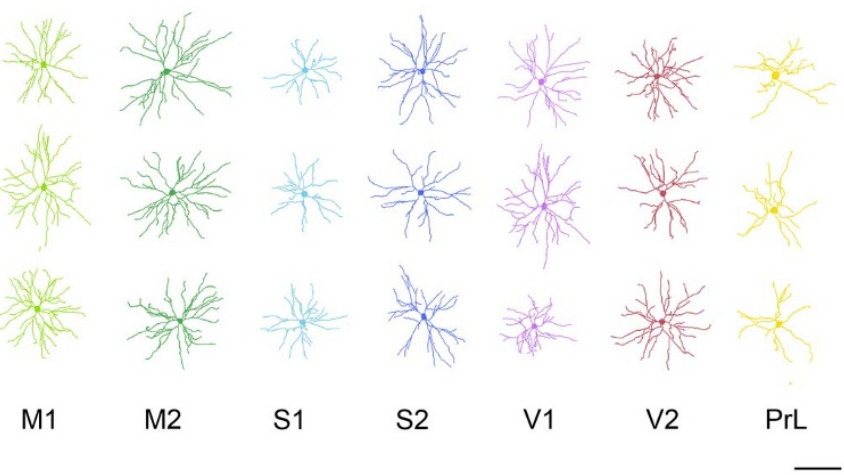

Figure 1 Examples of Neurolucida drawings of basal dendritic arbors of layer III pyramidal neurons, as seen in the plane of section parallel to the cortical surface, in the seven cortical areas analyzed: primary (M1) and secondary (M2) motor cortex, primary (S1) and secondary (S2) somatosensory cortex, primary (V1) and secondary (V2) visual cortex and prelimbic/infralimbic cortex $(\operatorname{PrL} / \mathrm{IL})\left(\right.$ modified from $\left.{ }^{30}\right)$. Neurons in different cortical areas are colored according to the color code used for Figure 4. Scale bar: $100 \mu \mathrm{m}$.

dendritic arbors of hundreds of intracellularly injected cortical pyramidal cells in different cortical regions of the mouse (Figure 1).

\section{Results}

In the present study, we specifically analyzed branching angles of a database of 345 3D reconstructed basal dendritic arbors of intracellularly injected layer III pyramidal neurons from seven different cortical areas of the frontal, parietal, and occipital cortex (see Materials and Methods for details). For this purpose, dendritic angles between two dendritic segments growing from a given bifurcation were grouped according to two different ordering schemes, based on the order in which the bifurcations occurred (see Figure 2A). These two ordering schemes were chosen in order to analyze angles from different perspectives. In the case of the standard order, the cell body is taken as the 'starting point' from which the bifurcations are progressively numbered 1,2,3, etc. By contrast, in the case of the inverse order, it is the distal end of the dendrites that is taken as the 'starting point' from which the bifurcations are progressively numbered - in this case as $1^{\prime}, 2^{\prime}, 3^{\prime}$, etc.

A visual inspection of the rose diagrams (Figure $2 \mathrm{~B}$ and Supplementary Figure S2 for all cortical areas examined) showed that the distributions of the angles were clearly unimodal and symmetric around the mean in almost all branch orders and cortical areas. Thus, we proposed modeling the bifurcation angles using the von Mises distribution ${ }^{29}$ and performed goodness-of-fit tests to check this hypothesis. We concluded that the von Mises distribution seems to be appropriate for modeling bifurcation angles of the same branching order in both standard and inverse branch order representations (see Supplementary Tables 2-5).

Comparison of angles of different branch orders. We first compared angles of different branch orders in general, according to the standard and inverse branch orders. Since classical linear boxplots are not suitable for conveying angular data, here we propose an adaptation of the boxplot for displaying the summary statistics computed for a dataset of angles, which we called circular boxplot (see Materials and Methods for further details). As shown in Figure 2C,D, angles tended to significantly decrease when standard branch order increases, whereas the opposite was found for inverse branch orders. This was the case for all cortical regions examined (Supplementary Figure S3, and Supplementary Tables 6-7 [standard] and 10-11 [inverse] for statistical significance results). Statistically significant differences are also seen in the test-based diagrams. First, motor (M1, M2) and somatosensory (S1, S2) areas behaved similarly for the standard branch order, since only orders 3 and 4 (out of the possible 6 pairwise comparisons) were not significantly different, as shown by the edge between nodes 3 and 4 in the test-based diagrams (Figure 2C,D and Supplementary Figure S3). Second, in the secondary visual cortex (V2) area, all standard orders were significantly different (there are no edges between nodes). Finally, the primary visual cortex (V1) and prelimbic/infralimbic cortex (PrL) had a statistically significant difference between orders 1 and 3 (in V1 also 2 and 3). In both areas, orders 1-2, 1-4, 2-4 and 3-4 (and 2-3 for PrL) had similar mean angles (there are edges connecting these pairs). Then, we compared angles of different branch orders originating from dendritic trees of similar complexity (i.e. different dendritic trees were grouped according to their maximum branch order, in each cortical region). This analysis (Figure 2E and Supplementary Figure S4 for all cortical areas examined, and Supplementary Tables 8-9 [standard] and 12-13 [inverse] for statistical results) showed even greater statistical differences between the branching angles of different orders. The test-based diagrams also show this difference, with no links between nodes except for the pair 3-4 (standard order) and 3'-4' (inverse). Thus, bifurcations nearer to the soma (lower standard branch orders) have wider angles than bifurcations at higher orders (with these differences being statistically significant). Accordingly, lower inverse branch orders have significantly narrower angles than higher inverse orders. This is clearly the case when angles are selectively grouped according to the maximum branch order of their dendritic trees.

Comparison of angles of the same branch order that belong to dendritic trees of different complexity. We then compared angles of the same branch order but coming from dendritic trees with different maximum branch order (i.e. order 1 angles that belong to dendritic trees of different complexity were then directly compared). This analysis (Figure 3 left, first row in Supplementary Table 14 and first block of rows in Supplementary Table-15) revealed that, for a particular cortical region, the standard branch order 1 angle is wider in dendritic trees that have higher orders, with the largest ones showing the most complex dendritic trees (statistical differences were only found in M1, M2, S1 and PrL, where some edges are absent in their test-based diagrams). We also found some statistically significant differences between angles at intermediate standard orders (2 and 3) for all cortical regions (see Supplementary Figure S5, second and third rows in Supplementary Table 14 and second and third blocks of rows in Supplementary Table-15). Thus, bifurcations at lower standard branch orders seem to require wide angles to accommodate the rest of the dendritic tree, suggesting that the dendritic trees grow to fill up the space defined by the lowest order angle (standard branch order 1).

By contrast, the inverse branch order 1' angle was similar for the majority of the dendritic trees of a particular region (except in V2), regardless of their complexity, as shown by the high connectivity between nodes in the test-based diagrams (see Figure 3 right, first row in Supplementary Table 16 and first block of rows in Supplementary Table 17). Also, intermediate inverse orders (2 and 3) were deemed not significantly different for the majority of the dendritic trees of a particular region, regardless of their complexity (see Supplementary Figure S5, second and third rows in Supplementary Table 16 and second and third blocks of rows in Supplementary Table 17).

Comparison between cortical areas. We finally compared angles, per branch order, between different cortical regions (Figure 4 and Supplementary Tables 18-21) and we found that the mean standard branch order 1 angle (Figure 4 top left) was rather similar in the various cortical regions (first column in Supplementary Table 18 and first block of rows in Supplementary Table 19). This is captured by the dense test-based diagram. By contrast, the mean inverse branch order $1^{\prime}$ angle (Figure 4 top right) was remarkably 

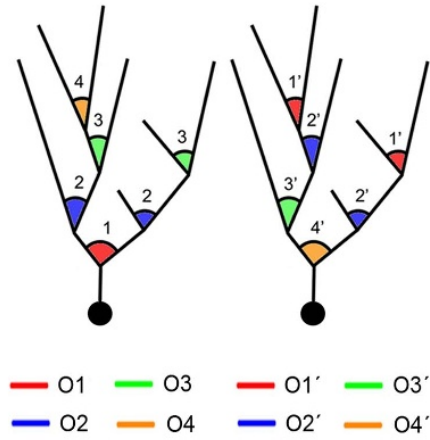

$\mathrm{E}$

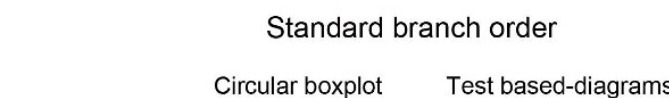

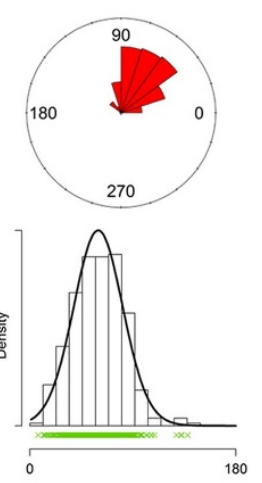

Standard branch order
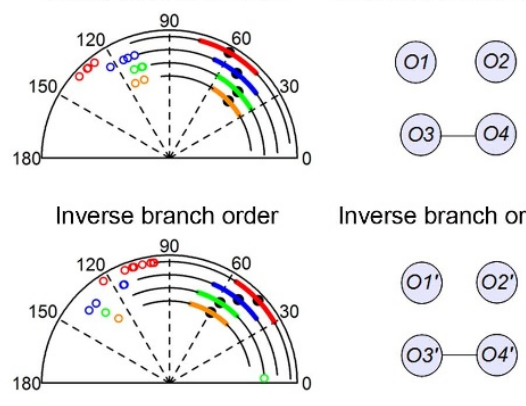

Inverse branch order

(01.)

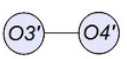

Inverse branch order

Circular boxplots

Test-based diagrams
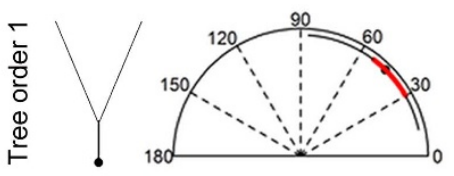

(01)

(O2)
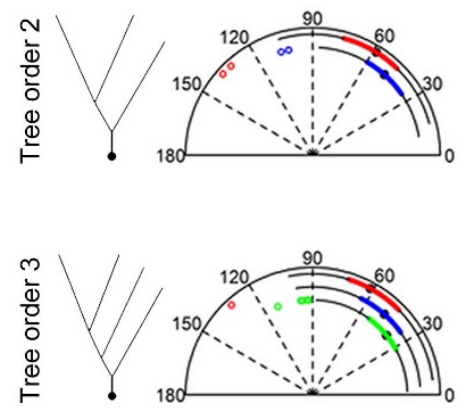

(01)

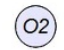

(03)

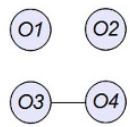

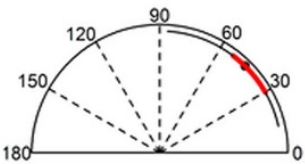

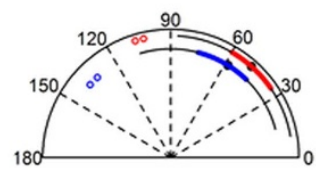

(01)
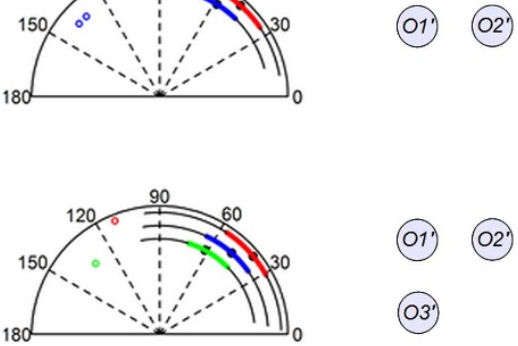

01

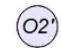

(03)

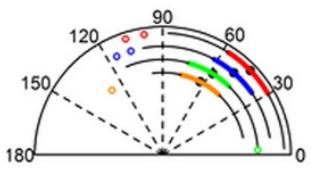

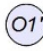

(03.) (04)

Figure 2 (A): Schematic showing the branching angles measured between sibling segments, according to two schemes: standard and inverse branch orders. (B): Example of the distribution of angles for standard branch order 1 coming from basal dendritic trees of pyramidal neurons in M2 area. The (upper) rose diagram shows the proportion of data in each interval of angles (in degrees). The (lower) density plot shows the empirical histogram of the dataset (bars) and the fitted von Mises density (thick black line). The dataset is shown with green crosses below the histogram. (C): The proposed circular boxplots of the angles showing the summary statistics of a dataset as arcs inside a circle, for standard (upper diagram) and inverse (lower diagram) branch orders. A black dot represents the median of the dataset, whereas the colored thick lines (same color code as in A) extend from the lower quartile (Q1) to the upper quartile $(\mathrm{Q} 3)$. The black lines extend from the minimum to the maximum values in the dataset included in the interval [Q1 - $1.5 \cdot \mathrm{IQR}, \mathrm{Q} 3+$ $1.5 \cdot \mathrm{IQR}]$, where IQR $=\mathrm{Q} 3-\mathrm{Q} 1$ is the interquartile range. The small colored dots indicate data values that are not in this interval, and are considered as outliers (see Supplementary Figure S1 for further details). (D): Test-based diagrams illustrating the pairwise comparisons of the mean angles from datasets shown in (C). Two nodes (representing two datasets) are connected when the hypothesis of equal mean angles of the corresponding datasets using Watson test cannot be rejected. Two nodes are not connected if the mean angles were deemed significantly different. See Supplementary Tables 6-7 (standard) and 10-11 (inverse) for further details on statistical tests. (E): Circular boxplots coming from basal dendritic trees of pyramidal neurons from M2 region, grouped according to their branch complexity (same color code as in A). Test-based diagrams are presented next to each graph to show comparison results between mean angles of different branch orders. See Supplementary Tables 8-9 and 12-13 for further details on statistical tests and Supplementary Figures S2-S4 for the corresponding diagrams of the remaining cortical regions examined (M1, S1, S2, V1, V2 and PrL). O1-O4: standard branch order $1-4 ; \mathrm{O}^{\prime}-\mathrm{O} 4{ }^{\prime}$ : inverse branch order $1-4$.

different in the seven cortical regions (first column in Supplementary Table 20 and-first block of rows in Supplementary Table 21). In particular, M1, M2 S1 and V2 showed smaller final bifurcation angles than V1, S2 and PrL. These areal features show that, in general, cortical regions with larger dendritic trees ${ }^{30}$ have smaller final bifurcation angles. 
Standard branch $\mathrm{O} 1$

M1

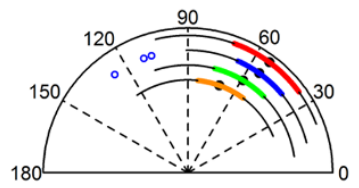

M2

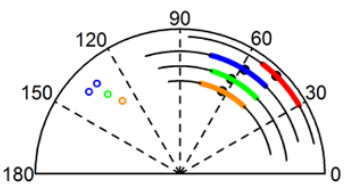

S1

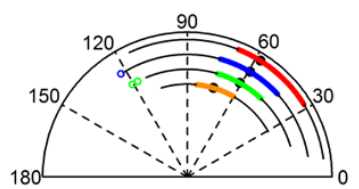

S2
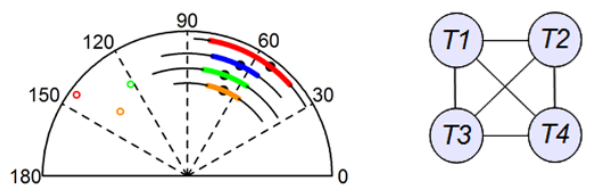

V1
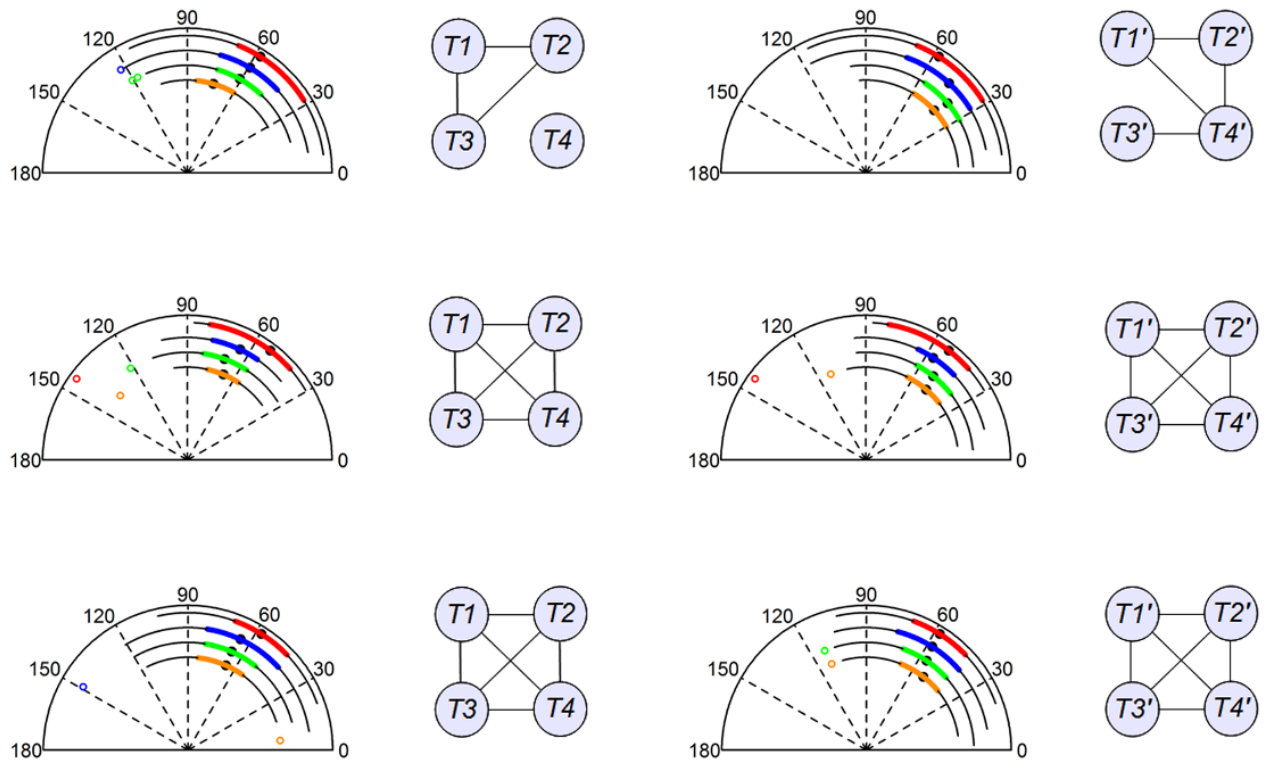

V2
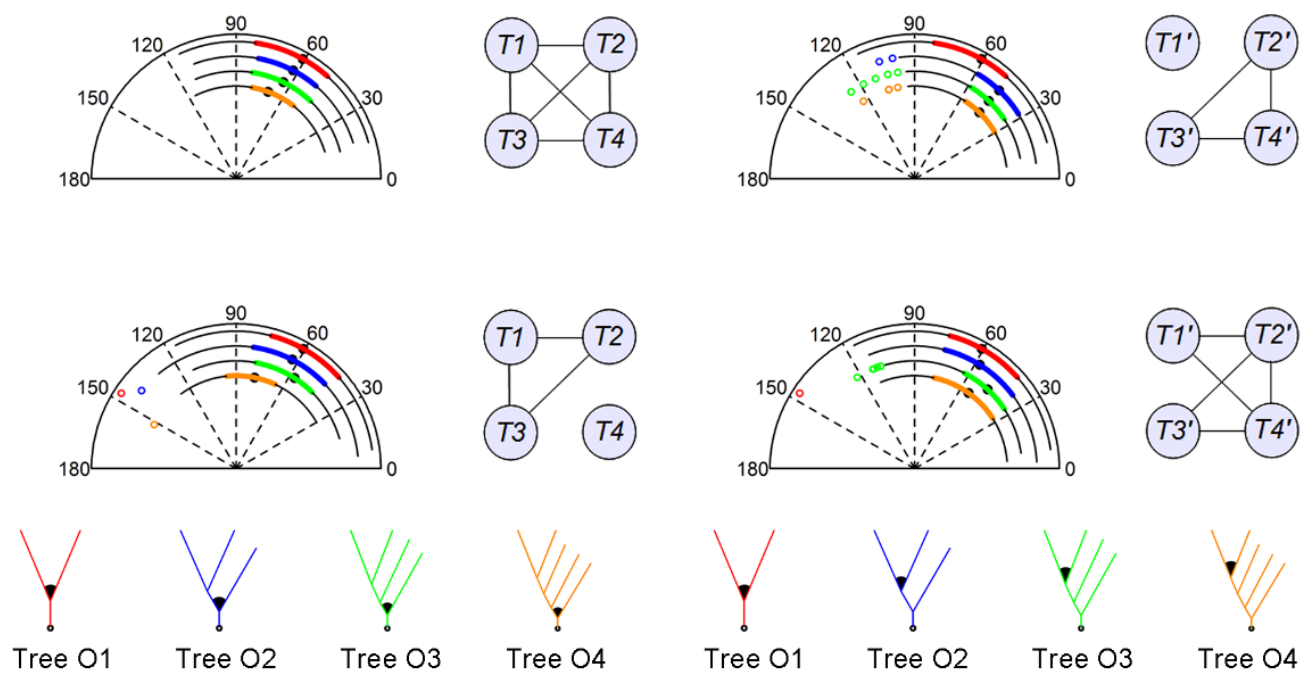

Figure 3 Circular boxplots showing comparison of angles of branch order 1 and 1' coming from dendritic trees with different maximum branch order. Mean values are shown per standard branch order (on the left) and inverse branch order (on the right) from basal dendritic trees of the seven cortical areas analyzed. Test-based diagrams illustrating the pairwise comparisons of the mean angles are shown next to each graph (nodes are denoted starting with a "T", which stands for "tree"). See Supplementary Tables 14-17 for further details on statistical tests. See Supplementary Figure S5 for the corresponding diagrams of the remaining branch order comparisons $(\mathrm{O} 2-\mathrm{O} 3)$. 


\section{Standard branch order}

01
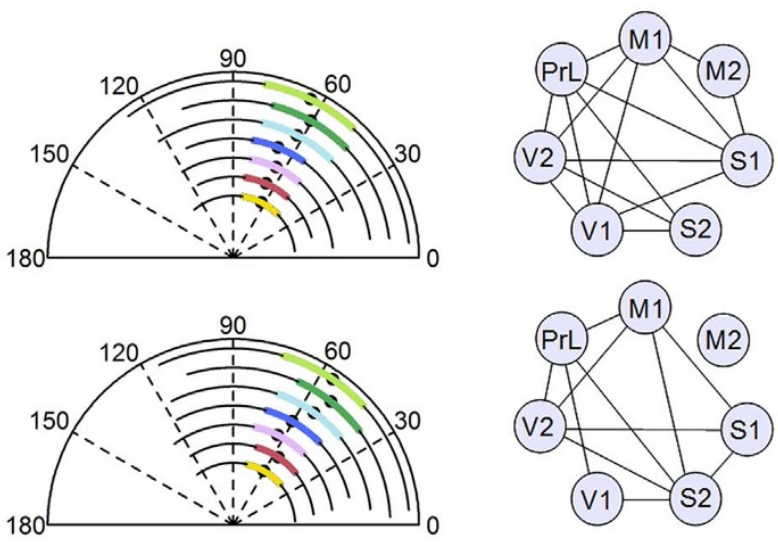

$\mathrm{O} 2$

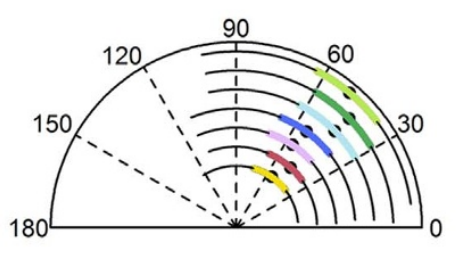

$\mathrm{O} 3$

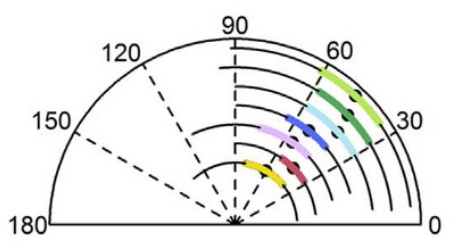

O4
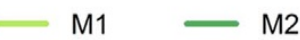

M2

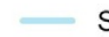

S1
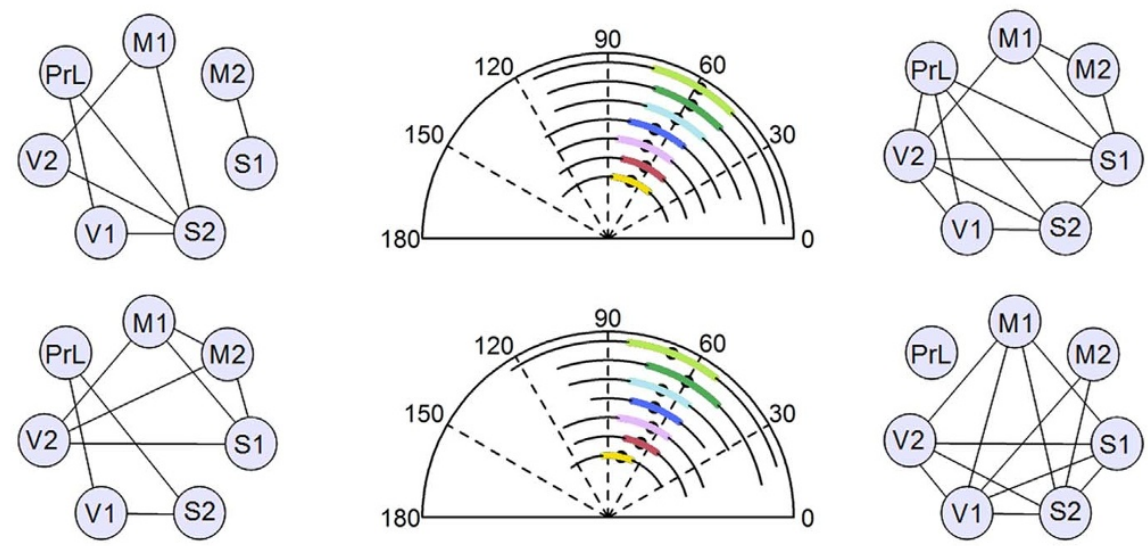

Figure 4 Circular boxplots showing the comparison of angles, by branch order, of the seven different cortical regions analyzed for standard branch order (left) and inverse branch order (right). Test-based diagrams are shown next to the corresponding boxplot. Node names are the cortical areas. (see Supplementary Tables 18-21 for further details on statistical tests).

\section{Discussion}

The main findings in the present study are the following. First, branching angles in pyramidal basal dendritic arbors can be modeled with von Mises distributions. Second, the first bifurcation (angle 1) of a particular basal tree is the widest and subsequent bifurcations become progressively narrower. Third, the final bifurcation (angle $1^{\prime}$ ) of a particular region is rather similar, regardless of the branch order of the dendrite. Finally, standard mean branch order 1 angle was rather similar in the various cortical regions whereas the mean inverse branch order $1^{\prime}$ angle was remarkably different in the seven cortical regions.

The first finding that a theoretical distribution (von Mises) was found for branching angles of pyramidal cells allows progress to be made in the area of dendritic structure modeling of the pyramidal cells in each cortical area. It demonstrates that these components of cortical circuits are designed in accordance with the rules of mathematical functions. Furthermore, $90 \%$ of these angles fell within a range of 20 to 97 degrees (per cortical area, mean angles ranged from 59 to 68 degrees and concentrations ranged from 5 to 8 degrees), suggesting certain predictability in the synaptic connections of pyramidal cells in all cortical areas. This is important since the vast majority of synapses on pyramidal neurons are established with their dendritic spines and the length of these spines is typically $<2 \mu \mathrm{m}$, (e.g. ${ }^{12,31}$ ). Thus, as a consequence of the restrictions conferred by such a short dendritic spine length, the rules that govern the dendritic branching of pyramidal cells can in fact determine the connectivity of the pyramidal cell.

The second geometrical rule found is that the first bifurcation (angle 1) of a particular tree is the widest and subsequent bifurcations become progressively narrower. Additionally, the final bifurcation (angle $1^{\prime}$ ) of a particular region is rather similar, regardless of the branch order of the dendrite. Hence, both 1 and $1^{\prime}$ angles would predict the final complexity of the dendritic tree for a particular region in such a way that, given the apparently predetermined amplitude of angle $1^{\prime}$, the wider the angle 1 , the greater the degree of branching of the tree. These results reveal that there are rules that govern the geometry of dendritic branching angles of pyramidal cells and that these rules follow common design principles in all cortical areas despite the different functional specializations. However, since the final bifurcation angle $1^{\prime}$ mean value was found to be statistically different between cortical regions, our findings suggest that the architecture of branching angles is also regulated regionally.

Finally, as reviewed $\mathrm{in}^{32}$, the process of dendrite formation is regulated at multiple points by a combination of cell-intrinsic and -extrinsic factors: first, the number of primary branches initiated and maintained in any one direction; second, the mode of branching; third, the frequency of branching; and fourth, the balance between growth, stabilization and retraction of dendritic branches (for a recent study, see ${ }^{33}$ ). Our findings are in line with the idea that genetic control is a strong determinant of the general shape of the pyramidal 
dendritic trees, whereas specific factors should be involved in the shaping of the fine details of the pyramidal cell morphology characteristic of each cortical area. Further, studies will be necessary to establish if changes in the complexity of the dendritic trees that are known to occur as a result of a variety of experimental factors (e.g..$^{31,34,35}$ ) are due to changes in branching angles. Similarly, in cases of brain diseases whose underlying anatomical changes have not been well established, such as autism and schizophrenia, determining whether or not these angles change could represent a major step for better understanding these diseases. The present results propose a new geometrical approach that could help clarify these issues by analyzing the rules of synaptic connections in the normal brain and under experimental or pathological conditions.

\section{Methods}

Data acquisition and preparation. A set of 3453 D reconstructed pyramidal neurons from seven different regions of the mouse neocortex was used for analysis. Pyramidal neurons were intracellularly injected with Lucifer Yellow in layer III horizontal sections to allow the complete inclusion of basal dendritic arbors. The regions analyzed included cortical areas of the frontal, parietal, and occipital cortex: primary (M1) and secondary (M2) motor cortex, primary (S1) and secondary (S2) somatosensory cortex, primary (V1) and secondary (V2) visual cortex and prelimbic/ infralimbic cortex (PrL) (see Ballesteros-Yáñez et al., 2010 for further methodological details). Reconstruction of the same neurons, using the Neurolucida package (MicroBrightField) has been used previously in another study for different purposes $^{30}$. We measured the angle between the two sibling segments originating from a bifurcation in the 3D reconstructions of the basal dendritic trees. Given a bifurcation point $\boldsymbol{O}$ with coordinates $\left(x_{o}, y_{o}, z_{o}\right)$, and two points $\boldsymbol{A}=\left(x_{a}, y_{a}, z_{a}\right)$ and $\boldsymbol{B}$ $=\left(x_{b}, y_{b}, z_{b}\right)$ defining the end points of the segments growing from the bifurcation (where the "end points" are either a terminal tip or another bifurcation point), the angle $\varphi$ between the vectors $O A$ and $O B$ is given by:

$$
\varphi=\arccos ((\boldsymbol{O A} \cdot \mathbf{O B}) /(\|\mathbf{O A}\|\|\mathbf{O B}\|))
$$

where $\cdot$ represents the scalar product of the vectors and $\|\boldsymbol{x}\|$ is the magnitude of the vector $\boldsymbol{x}$. Branch order angles higher than $\mathrm{O} 4$ were not included in the analysis due to their relatively low number (see Supplementary Table 1).

The von Mises distribution for angular data. Directional and angular data have some distinctive properties that make classical statistics unsuitable for analyzing and working with this kind of data. Directional statistics ${ }^{36-38}$ provides the theoretical background and the tools necessary for correctly managing these data. The von Mises (1918) distribution is the angular analog of the Gaussian distribution, and it is the most commonly used distribution in directional statistics. Different visualization tools from those used in classical linear statistics are necessary to convey directional information (e.g. the linear boxplot disregards the periodicity of the domain and wrongly estimates the summary statistics for the first dataset; see also Supplementary Figure S1). Thus, rose diagrams were used instead of regular histograms to represent the empirical distribution of a dataset of angles (Figure 2B). The domain of the von Mises density is the unit circle which defines angles $x \in[0,2 \pi]$. A random variable $X$ following a von Mises density with parameters $(\mu, \kappa)$ is denoted $M(\mu, \kappa)$. The von Mises distribution is a two-parameter distribution with probability density function:

$$
f(x, \mu, \kappa)=\frac{1}{2 \pi I_{0}(\kappa)} \exp (\kappa \cos (x-\mu))
$$

where $\mu$ is the mean angle of $X, \kappa \geq 0$ is the concentration of the angles around the mean and $I_{0}$ is the modified Bessel function of the first kind of order 0:

$$
I_{0}(\kappa)=\frac{1}{2 \pi} \int_{0}^{2 \pi} \exp (\kappa \cos x) d x
$$

The density of the points in the circle becomes uniform when $\kappa=0$, whereas high values of $\kappa$ yield points that are tightly clustered around $\mu$. The von Mises density is unimodal and symmetric around the mean direction. The mean direction is also the mode. The von Mises density is close to the Gaussian density when the concentration $\kappa$ is high and close to the Cauchy density when $\kappa$ is low.

Given a dataset with $N$ angles $\left\{x_{1}, \ldots, x_{N}\right\}$ randomly sampled from $M(\mu, \kappa)$, the maximum likelihood estimator of $\mu$ is the sample mean direction. On the other hand, the maximum likelihood estimator of $\kappa$ cannot be computed analytically and numerical approximations have to be used ${ }^{36,37}$. Here, the 'circular' package for $\mathrm{R}$ was used to compute the maximum likelihood estimators of the parameters. See some examples in Supplementary Figure S2, where a histogram and the fitted von Mises density are superimposed for data from the same cortical area and branch order.

Graphical representations of angular data. In this section, we describe the two graphical representations used in this study to portray the results of the analyses.
Rose diagrams. In a rose diagram, the circumference is divided into $K$ sectors with the same arc length and the area of each sector shows the number of angles contained in a given interval of angles. To achieve this, the radius of each sector needs to be proportional to the square root of the number of angles in the interval. In this study we used $K=20$ sectors. See some examples in Supplementary Figure S2.

Circular boxplots for angular data. We introduce here a circular analog of the wellknown boxplot, that we termed circular boxplot. The summary statistics of a dataset are shown as arcs inside a circle (Figure 2C). The median of the dataset is shown with a marked dot. The colored thick lines extend from the lower quartile (Q1) to the upper quartile (Q3) of the dataset. The whiskers (thin black lines) extend from the minimum to the maximum values in the dataset included in the interval $[\mathrm{Q} 1-1.5 \cdot \mathrm{IQR}, \mathrm{Q} 3+$ $1.5 \cdot \mathrm{IQR}]$, where $\mathrm{IQR}=\mathrm{Q} 3-\mathrm{Q} 1$ is the interquartile range. Data values outside this range are considered outliers and shown with small colored dots. This representation allows the boxplots of different datasets to be displayed in the same graph, by plotting the corresponding arcs at different distances from the circle's center. The angles at the bifurcations in basal dendrites have values from 0 to 180 degrees. Therefore, the circular boxplots were displayed over a semicircle instead of the complete circle (see Supplementary Figures S3-S5)

Statistical tests. Tests of goodness-of-fit to a von Mises distribution. We tested whether or not the datasets of bifurcation angles at different standard/inverse orders can be modeled with a von Mises density, $M(\mu, \kappa)$, with unknown parameters. We performed a Watson test for goodness-of-fit to a von Mises distribution ${ }^{39}$, using the quantiles computed $\mathrm{as} \mathrm{in}^{40}$. Based on these quantiles, the intervals for the $p$-value of each test are those reported in Supplementary Tables 2-5. A significance level $\alpha=0.05$ was used.

Analysis of variance for angular data. We looked for statistically significant differences between the mean angles of several datasets. Given a number $S$ of different datasets of angles, we applied the Watson-Williams test ${ }^{41,42}$ to check the null hypothesis of equal means in all the datasets, i.e., $\left(H_{0}: \mu_{1}=\mu_{2}=\cdots=\mu_{S}\right)$. The Watson-Williams test assumes that the $S$ datasets follow von Mises distributions (verified by our data) with equal concentrations. However, this test has proven to be robust against deviations from these assumptions ${ }^{43}$. See Supplementary Tables 6, 8, 10, 12, 14, 16, 18 and 20 .

Non-parametric tests for comparing the means of two datasets of angles. When the Watson-Williams tests found statistically significant differences, we performed all the pairwise comparisons between the $S$ datasets and looked for specific differences in their mean angles. We applied a non-parametric test to look for differences between two datasets without assuming a known generating parametric distribution. Watson tes ${ }^{44}$ was used to check the null hypothesis of equal means of the two datasets $\left(H_{0}: \mu_{1}\right.$ $=\mu_{2}$ ). We used a significance level $\alpha=0.05$. See Supplementary Tables 7, 9, 11, 13, 15, 17,19 and 21 .

Test-based diagrams. Here, we propose a novel way to show graphically the results of the Watson tests of all the pairwise comparisons between $S$ different datasets (see previous section). We built a graph with $S$ nodes, one for each dataset in the comparison. Two nodes were connected by an edge when we could not reject the null hypothesis of equal mean angles using Watson test. Thus, two nodes were not connected if the mean angles of the corresponding datasets were deemed significantly different. These statistical diagrams are shown in Figures 2D, 2E, 3 and 4 of the main text and Supplementary Figures S3-S5.

1. DeFelipe, J. \& Fariñas, I. The pyramidal neuron of the cerebral cortex: Morphological and chemical characteristics of the synaptic inputs. Progress in Neurobiol 39, 563-607 (1992).

2. White, E. Cortical circuits: Synaptic organization of the cerebral cortex. Structure, function and theory (Birkhauser, Boston, 1989).

3. Feldman, M. [Morphology of the neocortical pyramidal neuron] Cerebral cortex. Cellular components of the cerebral cortex [Peters, A. \& Jones, E. (Eds.)] [pp. 201-253] (Plenum Press, New York, 1984).

4. Bourne, J. N. \& Harris, K. M. Balancing structure and function at hippocampal dendritic spines. Annu Rev Neurosci 31, 47-67 (2008).

5. Yuste, R. \& Bonhoeffer, T. Genesis of dendritic spines: Insights from ultrastructural and imaging studies. Nature Rev Neurosci 5, 24-34 (2004).

6. Spruston, N. Pyramidal neurons: Dendritic structure and synaptic integration. Nature Reviews Neurosci 9, 206-221 (2008).

7. Elston, G. N. \& Rosa, M. G. The occipitoparietal pathway of the macaque monkey: comparison of pyramidal cell morphology in layer III of functionally related cortical visual areas. Cereb Cortex 7, 432-452 (1997)

8. Elston, G. N., Benavides-Piccione, R. \& DeFelipe, J. The pyramidal cell in cognition: a comparative study in human and monkey. J Neurosci 21, RC163 (2001).

9. Elston, G. N. et al. Specializations of the granular prefrontal cortex of primates: implications for cognitive processing. Anat Rec A Discov Mol Cell Evol Biol 288, 26-35 (2006).

10. Jacobs, B. et al. Regional dendritic and spine variation in human cerebral cortex: A quantitative Golgi study. Cereb Cortex 11, 558-571 (2001).

11. Benavides-Piccione, R., Hamzei-Sichani, F., Ballesteros-Yáñez, I., DeFelipe, J. \& Yuste, R. Dendritic size of pyramidal neurons differs among mouse cortical regions. Cereb Cortex 16, 990-1001 (2006). 
12. Ballesteros-Yáñez, I., Benavides-Piccione, R., Elston, G. N., Yuste, R. \& DeFelipe, J. Density and morphology of dendritic spines in mouse neocortex. Neurosci 138, 403-409 (2006).

13. Bianchi, S. et al. Dendritic Morphology of Pyramidal Neurons in the Chimpanzee Neocortex: Regional Specializations and Comparison to Humans. Cereb Cortex 23, 2429-2436 (2013).

14. Koch, C. Biophysics of computation. Information processing in single neurons (Oxford University Press, New York, 1999).

15. Mel, B. "Why have dendrites? A computational perspective" in: Dendrites, ed. Stuart, G., Spruston, N. \& Häusser, M. (pp. 271-289). New York, Oxford University Press (1999).

16. Chklovskii, D. B., Mel, B. W. \& Svoboda, K. Cortical rewiring and information storage. Nature 431, 782-788 (2004).

17. London, M. \& Häusser, M. Dendritic computation. Ann Rev Neurosci 28, 503-532 (2005).

18. Häusser, M., Spruston, N. \& Stuart, G. J. Diversity and dynamics of dendritic signaling. Science 290, 739-744. Review (2000).

19. López-Cruz, P. L., Bielza, C., Larrañaga, P., Benavides-Piccione, R. \& DeFelipe, J. Models and simulation of 3D neuronal dendritic trees using Bayesian networks. Neuroinformatics 9, 347-369 (2011).

20. Samsonovich, A. V. \& Ascoli, G. A. Statistical morphological analysis of hippocampal principal neurons indicates cell-specific repulsion of dendrites from their own cell. J Neurosci Res 71, 173-187 (2003).

21. Memelli, H., Torben-Nielsen, B. \& Kozloski, J. Self-referential forces are sufficient to explain different dendritic morphologies. Front Neuroinform 7, 1 (2013).

22. Cuntz, H., Borst, A. \& Segev, I. Optimization principles of dendritic structure. Theor Biol Med Model 4, 21(2008).

23. Cuntz, H., Forstner, F., Borst, A. \& Häusser, M. One rule to grow them all: A general theory of neuronal branching and its practical application. PLoS Comput Biol 6, e1000877 (2010).

24. Cuntz, H., Mathy, A. \& Häusser, M. A scaling law derived from optimal dendritic wiring. Proc Natl Acad Sci USA 109, 11014-11018 (2012).

25. Wen, Q. \& Chklovskii, D. B. A cost-benefit analysis of neuronal morphology. J Neurophysiol 99, 2320-8 (2008).

26. Wen, Q., Stepanyants, A., Elston, G. N., Grosberg, A. Y. \& Chklovskii, D. B. Maximization of the connectivity repertoire as a statistical principle governing the shapes of dendritic arbors. Proc Natl Acad Sci USA 106, 12536-41 (2009).

27. van Pelt, J. \& Uylings, H. B. The flatness of bifurcations in 3D dendritic trees: An optimal design. Front Comput Neurosci 5, 54 (2011).

28. Kim, Y1., Sinclair, R., Chindapol, N., Kaandorp, J. A. \& De Schutter, E. Geometric theory predicts bifurcations in minimal wiring cost trees in biology are flat. PLoS Comput Biol. 8, e1002474 (2012).

29. von Mises, R. Über die "Ganzzahligkeit" der Atomgewichte und verwandte Fragen. Physikalische Zeitschrift 19, 490-500 (1918).

30. Ballesteros-Yáñez, I., Benavides-Piccione, R., Bourgeois, J. P., Changeux, J. P. \& DeFelipe, J. Alterations of cortical pyramidal neurons in mice lacking highaffinity nicotinic receptors. Proc Natl Acad Sci USA 107, 11567-11572 (2010).

31. Benavides-Piccione, R., Fernaud-Espinosa, I., Robles, V., Yuste, R. \& DeFelipe, J. Age-Based Comparison of Human Dendritic Spine Structure Using Complete Three-Dimensional Reconstructions. Cereb Cortex 23, 1798-1810 (2012).

32. Landgraf, M. \& Evers, J. F. Control of dendritic diversity. Curr Opin Cell Biol. 17, 690-6 (2005).

33. Shimono, K., Fujishima, K., Nomura, T., Ohashi, M., Usui, T., Kengaku, M., Toyoda, A. \& Uemura, T. An evolutionarily conserved protein CHORD regulates scaling of dendritic arbors with body size. Sci Rep 4, 4415 (2014).
34. Jacobs, B., Schall, M. \& Scheibel, A. B. A quantitative dendritic analysis of Wernicke's area in humans. II. Gender, hemispheric, and environmental factors. J Comp Neurol 327, 97-111 (1993).

35. Fiala, J. C., Spacek, J. \& Harris, K. M. Dendritic spine pathology: cause or consequence of neurological disorders? Brain Res Rev 39, 29-54 (2002).

36. Fisher, N. I. Statistical Analysis of Circular Data (University Press, Cambridge 1993).

37. Mardia, K. V. \& Jupp, P. E. Directional Statistics (Wiley, London, 2000).

38. Jammalamadaka, S. R. \& SenGupta, A. Topics in Circular Statistics (World Scientific Publ, New jersey, 2001).

39. Watson, G. S. Goodness-of-fit tests on a circle. Biometrika 48, 109-114 (1961)

40. Lockhart, R. A. \& Stephens, M. A. Tests of fit for the von Mises distribution. Biometrika 72, 647-652 (1985)

41. Watson, G. S. \& Williams, E. On the construction of significance tests on the circle and the sphere. Biometrika 43, 344-352 (1956).

42. Stephens, M. A. Multi-sample tests for the Fisher distribution for directions. Biometrika 56, 169-181(1969).

43. Zar, J. H. Biostatistical Analysis (Prentice Hall Inc, New Jersey, 1999).

44. Watson, G. S. Statistics on Spheres [pp. 146-147] (Wiley, New York, 1983).

\section{Acknowledgments}

This work was supported by funding from the Spanish Ministry of Economy and Competitiveness (grants TIN2010-20900-C04-04 to C.B. and P.L. and BFU2012-34963 to J.DF.), CIBERNED (CB06/05/0066 to J.DF.), the Cajal Blue Brain Project, Spanish partner of the Blue Brain Project initiative from EPFL (to C.B., P.L. and J.DF.) and the Human Brain Project (to J.DF., C.B. and P.L.). R.B.-P. was supported by the Spanish Ministry of Economy and Competitiveness (CSIC).

\section{Author contributions}

J.D.F., P.L., C.B. and R.B.-P. designed research and P.L.-C. and R.B.-P. performed research and analyzed data. All authors wrote and reviewed the manuscript.

\section{Additional information}

Supplementary information accompanies this paper at http://www.nature.com/ scientificreports

Competing financial interests: The authors declare no competing financial interests.

How to cite this article: Bielza, C., Benavides-Piccione, R., López-Cruz, P., Larrañaga, P. \& DeFelipe, J. Branching angles of pyramidal cell dendrites follow common geometrical design principles in different cortical areas. Sci. Rep. 4, 5909; DOI:10.1038/srep05909 (2014).

This work is licensed under a Creative Commons Attribution-NonCommercialNoDerivs 4.0 International License. The images or other third party material in this article are included in the article's Creative Commons license, unless indicated otherwise in the credit line; if the material is not included under the Creative Commons license, users will need to obtain permission from the license holder in order to reproduce the material. To view a copy of this license, visit http:// creativecommons.org/licenses/by-nc-nd/4.0/ 